as we had the skills and expertise to resume the process and to find new undetected patients.

Our aim was to determine how well the newly implemented PLT Memory Pathway follows the standards outlined in the National Institute of Health \& Care Excellence (NICE) Clinical Guideline 97 (CG97): Assessment, management and support for people living with dementia and their carers.

Method. A retrospective analysis of all PLT referrals from July 2018 to February 2020 (20 months) was performed to identify patients on the community memory pathway and those with possible undetected cognitive impairment. Data were collected from electronic patient records which included demographics, primary and collateral history, cognitive testing and imaging, dementia type among others. Results were analysed using Microsoft Excel. Result. 41 patients were included (59\% female). $80 \%$ of patients were referred for memory problems or confusion. $63 \%$ had previous referrals to a memory service and was on the community memory pathway at the time of the referral. $34 \%$ were on anticholinergic medication but in only $14 \%$ were this documented as reviewed. $100 \%$ were offered and had head imaging. A finding worthy of note was the absence of any from the ethnic minority background. $63 \%$ of patients were given a memory diagnosis and $34 \%$ had anti-dementia medication started. Patients' families were made aware of the diagnosis in $83 \%$ of cases, due to the absence of next of kin details in the patient record. Primary Care was made aware in $100 \%$ of cases; post-diagnostic support was $100 \%$.

Conclusion. The PLT is well placed to bridge the service gap between the acute care trust and established community memory services when dealing with patients with dementia. A dedicated Memory Pathway has helped to close this gap and adherence to NICE CG97 standards was good, but there is room for improvement. A particular focus will be on improving documentation of anticholinergic medication review and exploration for the absence of ethnic minority patients. Aiming to achieve 100\% family involvement is also recommended.

This study has been submitted to the Royal College of Psychiatrists' Faculty of Old Age Annual Conference 2021.

\section{Addiction service changes due to COVID-19}

Shumaila Shahbaz ${ }^{1 \star}$, Zeeshan Hashmani ${ }^{2}$ and Soraya Mayet ${ }^{1}$

${ }^{1}$ Humber Teaching Foundation Trust and ${ }^{2}$ Tees Esk and Wear Valley NHS Foundation Trust

${ }^{*}$ Corresponding author.

doi: 10.1192/bjo.2021.914

Aims. Addictions services had to respond rapidly to reduce COVID-19 transmission to protect patients and staff. Patients with opioid dependence are particularly vulnerable, with high risks. Our community addiction service changed practice in line with COVID-19 guidelines. For patients with opioid dependence; face-to-face contacts were initially reduce and mainly for new starts, restarts and non-attenders. Prescribing changes were completed on an individually risk assessed basis to reduce attendance at the chemist, specifically to reduce transmission, keep patients in treatment and to ensure chemists could continue to function. We document some of the service changes during the COVID-19 lockdown.

Method. Service evaluation had approval from Humber Teaching NHS Foundation Trust. Data retrieved on one Hub of a community addictions service in North England, UK. Patients prescribed opioid substitution treatment for opioid dependence were assessed, with data retrieval through electronic healthcare records. Data were analysed by Microsoft Excel anonymously.

Result. In lockdown (March 2020 to June 2020), we identified 112 patients with opioid dependence prescribed opioid substitution (OST) with methadone or buprenorphine at the Hub. All white British, mean 42 years, most male (75\%) and prescribed methadone (78\%). Ten were new starts and 8 restarts to OST. Attendance rates did not change: $91 \%$ before and 92\% during lockdown. Appointment format changed from predominantly face-to-face $(92 \%)$ to telephone $(99 \%)$. Most patients $(91 \% ; n=$ 88) were offered take-home naloxone and overdose prevention training of which 14 refused. Supervision days at the chemist for OST reduced significantly from $75 \%$ collecting daily at the chemist, reducing to $20 \%$ during lockdown. Five patients were shielding and 7 had covid-related symptoms. There was one death during lockdown which was not attributed to covid or overdose.

Conclusion. The addictions service continued to be open and work proactively throughout lockdown, seeing new patients and continuing treatment interventions safely. Major changes were made in line with COVID-19 guidelines, to respond to the threat of transmission. Our service was flexible and able to adapt quickly to remote working. We maintained excellent attendance rates despite changes to the format of consultations. There were no related incidents e.g. overdoses linked to prescribed medications, despite a reduction in supervision, and therefore patients having extra medications. This important finding may be related to the individual risk assessments that we conducted before making changing to prescribing. This was supported by most patients were receiving naloxone to prevent overdoses. Some of the changes, such as telephone consultations, may be beneficial to continue post COVID-19.

\section{Audit of methods used to contact the duty doctor - Abraham Cowley Unit}

\author{
Ivan Shanley ${ }^{1 \star}$ and Jessica Thomas ${ }^{2}$ \\ ${ }^{1}$ Surrey and Borders Partnership NHS Foundation Trust and \\ ${ }^{2}$ Ashford and St Peter's NHS Foundation Trust \\ ${ }^{*}$ Corresponding author.
}

doi: 10.1192/bjo.2021.916

Aims. The aim of this audit was to determine whether the duty doctor of a 4 ward inpatient psychiatric unit is contacted safely, effectively and in a manner that can be monitored. This is in line with trust protocol and the method stated is via switchboard. Should a deficit be found it was the aim to make an appropriate intervention.

Background. In the Abraham Cowley Unit, there is a Senior House Officer 'on-call' duty doctor 24/7. The shifts are $2 \times 12.5$ hours daily and at all times the duty doctor should be contacted via switchboard. Contacting via switchboard is important to ensure there is an audit trail of calls made. Issues that arise from using other methods of contact, e.g. calling direct extensions, include miscommunication and the doctor not being reached in a timely manner. This had been identified as an issue anecdotally by junior doctors on call and also highlighted following an untoward incident.

Method. The method by which the on call doctor was contacted was recorded in Excel for 5 consecutive 12.5 hour shifts in October 2019. The standard set for calls via switchboard was $80 \%$. Following the initial results and the subsequent intervention, a repeat audit was performed using the same method. 